\title{
A New Theranostic Paradigm for Advanced Thyroid Cancer
}

\author{
David A. Pattison ${ }^{1,2}$, Benjamin Solomon ${ }^{3,4}$, and Rodney J. Hicks ${ }^{1,4}$ \\ ${ }^{1}$ Centre for Cancer Imaging, Peter MacCallum Cancer Centre, Melbourne, Victoria, Australia; ${ }^{2}$ Endocrinology Service, Peter MacCallum \\ Cancer Centre, Melbourne, Victoria, Australia; ${ }^{3}$ Department of Medical Oncology, Peter MacCallum Cancer Centre, Melbourne, Victoria, \\ Australia; and ${ }^{4}$ Sir Peter MacCallum Department of Oncology, University of Melbourne, Parkville, Victoria, Australia
}

$\mathbf{T}$ he successful use of ${ }^{131} \mathrm{I}$ as a systemic treatment of a patient with metastatic thyroid cancer was first reported in 1948 (1). This agent has subsequently become firmly established as part of the treatment of high-risk thyroid cancer and especially for metastatic disease. There has been an evolution in the quality of assessment of the distribution of radioactive iodine (RAI) within the body, progressing from Geiger-Müller counting to imaging, first with the rectilinear scanning and then with the $\gamma$-camera. The physical characteristics of ${ }^{123} \mathrm{I}$ as a diagnostic tracer compared with ${ }^{131} \mathrm{I}$ further improved imaging by facilitating higher quality SPECT and SPECT/CT. More recently, use of ${ }^{124}$ I PET/CT has further increased the sensitivity for detection of differentiated thyroid carcinoma (DTC) and offers the promise of prospective dosimetry estimation (2). Uptake of all these forms of RAI relies on the presence of functional sodium-iodide symporters, which are required for efficacious treatment with ${ }^{131} \mathrm{I}$.

\section{See page 1512}

Augmenting thyroid-stimulating hormone levels by combined use of thyroid resection and either withholding thyroid hormone or using recombinant human thyroid-stimulating hormone to stimulate RAI uptake has further improved the diagnostic paradigm of DTC, as has preparation of patients using a low-iodine diet and avoidance of iodinecontaining radiographic contrast agents or drugs, for example, amiodarone. Consequently, the proportion of truly non-RAI-avid disease has diminished. The role of ${ }^{18} \mathrm{~F}$-FDG PET/CT is now well established for detection and staging of disease in the context of abnormal structural imaging or elevated serum thyroglobulin (generally $>10 \mathrm{ng} / \mathrm{mL}$ ) but negative radioiodine imaging (3). The combined use ${ }^{18} \mathrm{~F}-\mathrm{FDG}$ PET/CT in appropriate cases has reduced the role of empiric ${ }^{131} \mathrm{I}$ therapy, particularly by localizing regional disease amenable to salvage surgery or radiotherapy. In some cases of metastatic disease, disease heterogeneity can be demonstrated with lesions with the highest ${ }^{18} \mathrm{~F}$ FDG uptake tending to be poorly differentiated as evidenced by loss of avidity on iodine imaging (4). Better localization and characterization of disease sites using these complementary techniques allows more rational selection and monitoring of patients with metastatic disease (5).

Received Mar. 2, 2016; revision accepted Mar. 2, 2016.

For correspondence and reprints contact: Rodney J. Hicks, Peter MacCallum

Cancer Centre, 12 Cathedral Place, Melbourne, Victoria 3002, Australia.

E-mail: rod.hicks@petermac.org

Published online Mar. 31, 2016

COPYRIGHT (C) 2016 by the Society of Nuclear Medicine and Molecular Imaging, Inc.

DOI: $10.2967 /$ jnumed.116.173534
Differentiation status and metabolic reprogramming are increasingly being recognized as determinants of imaging phenotype. In contrast to well-differentiated tumors, which are reliant on mitochondrial oxidative phosphorylation to generate the energy needed for cellular processes, poorly differentiated cancer cells depend on the inefficient mechanism of aerobic glycolysis - the Warburg effect. Notably, Hurthle cell (or oncocytic) tumors, both benign and malignant, are generally characterized by intense ${ }^{18} \mathrm{~F}$-FDG avidity representing inherent constitutive activation of glycolytic pathways (6). Loss of the mitochondrial respiratory chain complex I has been shown to be a marker of this oncocytic phenotype (7). Further phenotypic differences are reflective of genomic changes associated with neoplastic transformation, particularly including mutations involving the mitogenactivated protein kinase pathway. These provide potential therapeutic targets for non-iodine-avid thyroid cancer. The multikinase inhibitors lenvatanib (8) and sorafenib (9) have recently demonstrated significant improvement in progression-free survival in phase III trials of progressive radioiodine refractory disease, albeit with significant toxicities. Ho et al. (10) pioneered redifferentiation therapy in advanced thyroid cancer using a 4-wk course of the MEK inhibitor selumetenib to restore expression of sodium-iodide symporter, allowing ${ }^{131}$ I therapy, particularly in patients with NRAS mutations. There is favorable early data from patients with advanced thyroid cancer and $\mathrm{BRAF}^{\mathrm{V} 600 \mathrm{E}}$ mutations treated with BRAF inhibitors such as vemurafenib (11). The Cancer Genome Atlas Research Network's reclassification of papillary thyroid cancer into $\mathrm{BRAF}^{\mathrm{V} 600 \mathrm{E}}$-like or RAS-like molecular subtypes may potentially be used to stratify management of advanced thyroid cancer in the future (12).

This dual-tracer approach seems to address most cases of DTC. However, the article by Binse et al. in this issue of The Journal of Nuclear Medicine (13) addresses a small subgroup in which this paradigm still fails to identify a reason for persisting thyroglobulin elevation. In such cases, it might be assumed that the volume of disease is below the limits of imaging detection. However, Binse et al. challenge this idea by demonstrating the utility of somatostatin receptor (SSTR) imaging to detect previously occult disease. Their small consecutive series identified that uptake of ${ }^{68} \mathrm{Ga}$-DOTATOC, which targets SSTR subtypes 2 and 5, was most prevalent in patients with poorly differentiated or oncocytic carcinomas (4/4), compared with papillary (1/5) or follicular (0/6) thyroid cancers. Notwithstanding the limitations inherent in a retrospective study, including inconsistent RAI imaging methodology and timing, these findings are intriguing because poorly differentiated gastero-entero-pancreatic neuroendocrine tumors typically lose SSTR expression (14). The exception to this rule is insulinoma, in which a significant proportion of benign insulinoma (approximately one third) lack SSTR (15), whereas it is maintained in most malignant lesions, making it a suitable target for peptide receptor radionuclide therapy (PRRT) $(16,17)$. Unlike in most gastero-entero-pancreatic neuroendocrine tumors, a 


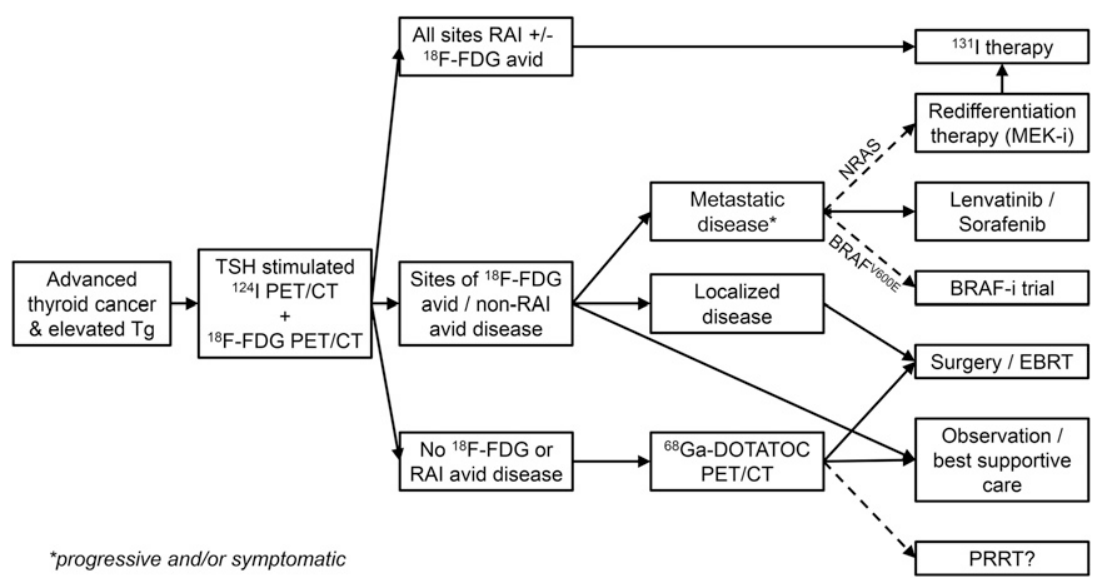

FIGURE 1. Proposed theranostic paradigm for advanced thyroid cancer using dual-tracer imaging with thyroid-stimulating hormone-stimulated ${ }^{124} \mathrm{I}$ and ${ }^{18} \mathrm{~F}$-FDG PET/CT to direct subsequent management, including ${ }^{68} \mathrm{Ga}-\mathrm{DOTATOC}$ PET/CT in those cases with nonlocalized disease despite elevated thyroglobulin. BRAF-I = BRAF inhibitor; EBRT = external-beam radiotherapy; $\mathrm{MEK}-\mathrm{I}=\mathrm{MEK}$ inhibitor; $\mathrm{RAI}=$ radioiodine; $\mathrm{TG}=$ thyroglobulin; $\mathrm{TSH}=$ thyroidstimulating hormone.

prior immunohistochemical study in thyroid malignancy demonstrated predominant expression of SSTR-5 rather than SSTR-2 (18), favoring radioligands with affinity for SSTR-5 (e.g., ${ }^{68} \mathrm{Ga}-\mathrm{DOTA}-$ NOC or ${ }^{68} \mathrm{Ga}$-DOTATOC) over SSTR-2 ligands (e.g., ${ }^{68} \mathrm{Ga}$-DOTATATE) in this disease. Hurthle cell carcinoma has been previously reported as having increased SSTR expression, comprising most cases in a previous small series treated with PRRT (19). Although localization of disease in $33 \%$ of cases in the current study provides the opportunity for additional local therapy, the relatively modest intensity of uptake identified within this small series (mean $\mathrm{SUV}_{\max }, 4.8$; range, 3.0-10.1) suggests that only a few cases will demonstrate sufficient uptake to enable PRRT.

Although it is not uncommon for poorly differentiated or oncocytic thyroid cancer to be non-RAI-avid, these subtypes typically demonstrate the greatest ${ }^{18} \mathrm{~F}-\mathrm{FDG}$ avidity. For example, a previously reported comparison of ${ }^{18} \mathrm{~F}-\mathrm{FDG}$ and ${ }^{68} \mathrm{Ga}$-DOTATOC in 17 patients with 104 lesions demonstrated that loss of SSTR expression coincided with a loss of iodine uptake (20). Although there was a similar incidence of ${ }^{18} \mathrm{~F}$-FDG and ${ }^{68} \mathrm{Ga}$-DOTATOC uptake in iodine-avid lesions, as expected, ${ }^{18} \mathrm{~F}$-FDG performed significantly better than ${ }^{68} \mathrm{Ga}$-DOTATOC in the 73 non-iodine-avid cohort, making the findings of the current study even more unusual.

In addition to a need to verify these findings in larger prospective studies, it will be important to better understand the biologic basis for SSTR expression in the thyroid and its role in cell signaling. This study certainly broadens the potential role of ${ }^{68} \mathrm{Ga}$-DOTATOC PET/CT to include cases of thyroid cancer with elevated thyroglobulin despite negative RAI and ${ }^{18} \mathrm{~F}-\mathrm{FDG}$ PET/CT. It also provides a unique opportunity to review the current role of molecular imaging in advanced thyroid cancer and outline a new theranostic paradigm incorporating SSTR imaging for this heterogeneous disease (Fig. 1).

\section{REFERENCES}

1. Seidlin SM, Oshry E, Yalow AA. Spontaneous and experimentally induced uptake of radioactive iodine in metastases from thyroid carcinoma: a preliminary report. J Clin Endocrinol Metab. 1948;8:423-432.

2. Wierts R, Brans B, Havekes B, et al. Dose-response relationship in differentiated thyroid cancer patients undergoing radioiodine treatment assessed by means of ${ }^{124}$ I PET/CT. J Nucl Med. February 25, 2016 [Epub ahead of print].

3. Haugen BR, Alexander EK, Bible KC, et al. 2015 American Thyroid Association management guidelines for adult patients with thyroid nodules and differentiated thyroid cancer: the American Thyroid Association Guidelines Task Force on Thyroid Nodules and Differentiated Thyroid Cancer. Thyroid. 2016;26:1-133.

4. Feine U, Lietzenmayer R, Hanke JP, Wohrle H, MullerSchauenburg W. ${ }^{18}$ FDG whole-body PET in differentiated thyroid carcinoma: flipflop in uptake patterns of ${ }^{18} \mathrm{FDG}$ and ${ }^{131} \mathrm{I}$ [in German]. Nuklearmedizin. 1995;34:127-134.

5. Pattison DA, Hofman MS. Role of fluorodeoxyglucose $\mathrm{PET} /$ computed tomography in targeted radionuclide therapy for endocrine malignancies. PET Clin. 2015; 10:461-476.

6. Zandieh S, Pokieser W, Knoll P, Sonneck-Koenne C, Kudlacek M, Mirzaei S. Oncocytic adenomas of thyroid-mimicking benign or metastatic disease on ${ }^{18}$ F-FDG-PET scan. Acta Radiol. 2015;56:709-713.

7. Gasparre G, Porcelli AM, Bonora E, et al. Disruptive mitochondrial DNA mutations in complex I subunits are markers of oncocytic phenotype in thyroid tumors. Proc Natl Acad Sci USA. 2007;104:9001-9006.

8. Schlumberger M, Tahara M, Wirth LJ. Lenvatinib in radioiodine-refractory thyroid cancer. N Engl J Med. 2015;372:1868.

9. Brose MS, Nutting CM, Jarzab B, et al. Sorafenib in radioactive iodine-refractory, locally advanced or metastatic differentiated thyroid cancer: a randomised, doubleblind, phase 3 trial. Lancet. 2014;384:319-328.

10. Ho AL, Grewal RK, Leboeuf R, et al. Selumetinib-enhanced radioiodine uptake in advanced thyroid cancer. $N$ Engl J Med. 2013;368:623-632.

11. Dadu R, Shah K, Busaidy NL, et al. Efficacy and tolerability of vemurafenib in patients with BRAF(V600E)-positive papillary thyroid cancer: M.D. Anderson Cancer Center off label experience. J Clin Endocrinol Metab. 2015;100:E77-E81.

12. Cancer Genome Atlas Research Network. Integrated genomic characterization of papillary thyroid carcinoma. Cell. 2014;159:676-690.

13. Binse I, Poeppel TD, Ruhlmann M, et al. ${ }^{68} \mathrm{Ga}$-DOTATOC PET/CT in patients with iodine- and ${ }^{18} \mathrm{~F}-\mathrm{FDG}-$ negative differentiated thyroid carcinoma and elevated serum thyroglobulin. J Nucl Med. 2016;57:1512-1517.

14. Binderup T, Knigge U, Loft A, Federspiel B, Kjaer A. ${ }^{18}$ F-fluorodeoxyglucose positron emission tomography predicts survival of patients with neuroendocrine tumors. Clin Cancer Res. 2010;16:978-985.

15. Reubi JC, Waser B. Concomitant expression of several peptide receptors in neuroendocrine tumours: molecular basis for in vivo multireceptor tumour targeting. Eur J Nucl Med Mol Imaging. 2003;30:781-793.

16. Wild D, Christ E, Caplin ME, et al. Glucagon-like peptide-1 versus somatostatin receptor targeting reveals 2 distinct forms of malignant insulinomas. J Nucl Med. 2011;52:1073-1078.

17. van Schaik E, van Vliet EI, Feelders RA, et al. Improved control of severe hypoglycemia in patients with malignant insulinomas by peptide receptor radionuclide therapy. J Clin Endocrinol Metab. 2011;96:3381-3389.

18. Woelfl S, Bogner S, Huber H, et al. Expression of somatostatin receptor subtype 2 and subtype 5 in thyroid malignancies. Nuklearmedizin. 2014;53:179-185.

19. Teunissen JJ, Kwekkeboom DJ, Kooij PP, Bakker WH, Krenning EP. Peptide receptor radionuclide therapy for non-radioiodine-avid differentiated thyroid carcinoma. J Nucl Med. 2005;46(suppl 1):107S-114S.

20. Middendorp M, Selkinski I, Happel C, Kranert WT, Grunwald F. Comparison of positron emission tomography with $\left[{ }^{18} \mathrm{~F}\right] \mathrm{FDG}$ and $\left[{ }^{68} \mathrm{Ga}\right] \mathrm{DOTATOC}$ in recurrent differentiated thyroid cancer: preliminary data. Q J Nucl Med Mol Imaging. 2010;54:76-83. 